\title{
Structure of cadmium sulfide nanoparticle micelle in aqueous solutions*
}

\author{
A. A. Rempel' ${ }^{a, b \star}$ N. S. Kozhevnikova, ${ }^{a, b}$ and S. V. Rempel ${ }^{\prime a, b}$ \\ ${ }^{a}$ Institute of Solid State Chemistry, Ural Branch of the Russian Academy of Sciences, \\ 91 ul. Pervomaiskaya, 620041 Ekaterinburg, Russian Federation. \\ E-mail: rempel@ihim.uran.ru \\ ${ }^{b}$ B. N. Eltsin Ural Federal University, \\ 19 ul. Mira, 620002 Ekaterinburg, Russian Federation
}

\begin{abstract}
The structure of cadmium sulfide (CdS) micelle in stable aqueous solution of ethylenediaminetetraacetic acid was determined by dynamic light scattering, small-angle X-ray scattering and neutron scattering. The micelle aggregate is a single CdS nanoparticle with an average size of about $3 \mathrm{~nm}$, the nanoparticle organic shell and the solvation shell are about $1 \mathrm{~nm}$ and $5 \mathrm{~nm}$ thick, respectively. These parameters were confirmed by the scanning semi-contact atomic force microscopy and powder X-ray diffraction studies of dry micelle cores isolated by highspeed centrifugation. The CdS micelle was correctly described by a simple double-shell model and was found to possess the structure corresponding to CdS quantum dots.
\end{abstract}

Key words: cadmium sulfide, chemical condensation from aqueous solutions, micelle structure.

Cadmium sulfide (CdS) nanoparticles and quantum dots (QD) are intensively studied because they possess unique catalytic and luminescent properties. ${ }^{1-4}$ Cadmium sulfide nanoparticles which are slightly larger in size than the exciton Bohr radius ( $3 \mathrm{~nm}$ ) can be used in catalysis for production of hydrogen, ${ }^{\mathbf{5 , 6}}$ for the purification of water from organic impurities, ${ }^{7,8}$ and in organic synthesis. ${ }^{9}$ Cadmium sulfide nanoparticles whose size is equal or smaller than the exciton Bohr radius in bulk CdS, i.e., CdS QD, are a promising material for the design of robust fluorescent biosensors, ${ }^{10,11}$ solar cells, ${ }^{12}$ and lasers. ${ }^{13}$

In the last twenty-five years, procedures for the synthesis of nanoparticles and colloidal QD were developed for many chalcogenides. The results on the synthesis of CdS nanoparticles and QD with various sizes and properties can be found in the literature. ${ }^{14-21}$ The main restraining factor for the fundamental studies and practical application of CdS nanoparticles is the toxicity of both cadmium ions ${ }^{22,23}$ and organic compounds used presently for the synthesis and stabilization of nanoparticles. ${ }^{17,20}$ Replacement of cadmium by nontoxic zinc, though being attractive for environmental reasons, has a fundamental disadvantage related to a too wide forbidden band of zinc sulfide $(3.68 \mathrm{eV})$; the blue shift can only broaden it. ${ }^{24}$ This leads to a shift of the absorption band to the UV region. Therefore, QD based on zinc sulfide cannot be used in many topical applications dealing with biology, medicine,

* Dedicated to Academician of the Russian Academy of Sciences S. M. Aldoshin on the occasion of his 60 th birthday. and visible-light photocatalysis. Replacement of CdS by silver and tin sulfides having narrower forbidden band is principally possible, but requires conducting of expensive preliminary quests and searches. However, all the alternative metals considered do not solve another important problem, viz., the toxicity of the organic stabilizing media.

A possible way to solve this problem is to synthesize nanoparticles in nontoxic aqueous media. However, CdS is a hydrophobic compound; therefore, its synthesis directly in the aqueous solution is accompanied by intense agglomeration. These processes lead not only to coagulation (sticking together), but also coalescence (a multiple increase in size) of nanoparticles. Only recently, ${ }^{25}$ a stable aqueous cadmium sulfide solution was obtained for the first time.

In the present work, we for the first time used modern methods for the structural studies of CdS micelles and made suggestions on the mechanism of their long-term stability against aggregation and sedimentation.

\section{Experimental}

Micelles studied in this work were obtained by chemical condensation following a known procedure. ${ }^{25}$ This method allows one to prepare stable aqueous colloidal solutions of $\mathrm{CdS}$ nanoparticles without using toxic organic solvents and makes it possible to avoide additional laborious manipulations related to the solubilization.

The micelles were synthesized from sodium sulfide $\mathrm{Na}_{2} \mathrm{~S}$, cadmium chloride $\mathrm{CdCl}_{2}$, and ethylenediaminetetraacetic acid

Published in Russian in Izvestiya Akademii Nauk. Seriya Khimicheskaya, No. 2, pp. 0400-0404, February, 2013. 
(EDTA, $\mathrm{C}_{10} \mathrm{H}_{16} \mathrm{~N}_{2} \mathrm{O}_{8}, \mathrm{H}_{4}$ edta). Reaction mixtures were prepared based on a $0.05 M$ aqueous solutions of $\mathrm{Na}_{2} \mathrm{~S}$ and $\mathrm{CdCl}_{2}$ and a $0.05 \mathrm{M}$ aqueous solution of EDTA disodium salt $\left(\mathrm{C}_{10} \mathrm{H}_{14} \mathrm{~N}_{2} \mathrm{O}_{8} \mathrm{Na}_{2}, \mathrm{Na}_{2} \mathrm{H}_{2}\right.$ edta $)$. The solutions were mixed in specified $\mathrm{v} / \mathrm{v}$ ratio at room temperature under continuous stirring. In mixing the starting solutions, the $\mathrm{Cd}^{2+}: \mathrm{S}^{2-}: \mathrm{H}_{2} \mathrm{edta}^{2-}$ concentration ratio was equal to $1: 1: 1$.

In the first step, a solution of the complex salt was obtained

$$
\mathrm{Cd}^{2+}{ }_{a q}+\mathrm{H}_{2} \mathrm{edta}^{2-}{ }_{a q}=\mathrm{Cdedta}^{2-}{ }_{a q}+2 \mathrm{H}^{+}{ }_{a q} .
$$

Then a solution of sulfidizing agent was added under continuous stirring

$$
\mathrm{Cdedta}^{2-}{ }_{\mathrm{aq}}+\mathrm{S}^{2-}{ }_{\mathrm{aq}} \rightleftharpoons \mathrm{CdS} \downarrow+\mathrm{edta}^{4-}{ }_{\mathrm{aq}} .
$$

Stable micelles were prepared at the starting $\mathrm{Cd}^{2+}, \mathrm{S}^{2-}$, and $\mathrm{H}_{2} \mathrm{edta}^{2-}$ concentrations in the reaction mixtures equal to $12.5 \mathrm{mmol} \mathrm{L}^{-1}$. The experiments carried out in the present work showed that the dilution of the stable solutions thus synthesized by a factor of up to 10 produced no changes in the size of the CdS nanoparticles. The dispersed CdS phase was isolated by highspeed centrifugation (an acceleration of $50000 \mathrm{~g}$ ) and dried in air at room temperature.

The sizes of the dispersed phase nanoparticles were determined by different methods directly in the aqueous solution after the synthesis ("moist" nanoparticles) and after centrifugation and/ or drying ("dry" nanoparticles). The refractive index of solutions required for the nanoparticle size measurements by optical methods was determined on an IRF-22 Abbe refractometer; its value was 1.33 .

A He-Ne laser operating at $633 \mathrm{~nm}$ was used in the dynamic light scattering (DLS) studies of micelles directly in the solution; the detector of the scattered radiation was placed at an angle of $173^{\circ}$. The studies of the size distribution function of the scattering centers showed that most micelles in the solution have a size of about $15 \mathrm{~nm}$ (Fig. 1). The measured zeta-potential of nanoparticles varied in the range from -75 to $-20.5 \mathrm{mV}$, thus indicating a rather high stability of the solution or, in other words, a low extent of coagulation.

High contrast between the electron density of the CdS nanoparticles and the electron density of the organic shell, as well as between the electron density of the dispersed phase and the dispersion medium allowed us to use the small-angle X-ray scattering (SAXS) for the determination of the diameter of the CdS micelle aggregate, which was found to be equal to $\sim 3 \mathrm{~nm} .{ }^{26}$ Using small-angle neutron scattering (SANS) and high contrast between the scattering cross-section of the organic shell and the scattering cross-section of the dispersion medium (heavy water was used as the solvent), we succeeded in rather accurate determining the thickness of the organic shell $(\sim 1 \mathrm{~nm})$ and confirming the diameter of the CdS nanoparticle determined by SAXS.

After centrifugation, dry nanoparticles were visualized by semi-contact atomic force microscopy (sc-AFM). An analysis of the surface relief measured by this method (Fig. 2) showed that the support is covered with small, round nanoparticles, among which large particles are rare to be found. The average size of small nanoparticles and the root-mean-square deviation determined from the results of 750 measurements were 2.9 and $1.3 \mathrm{~nm}$, respectively. ${ }^{27}$ The histogram of the nanoparticle size distribution (see Fig. 1) was approximated by a normal distribution function with the maximum at $2.6 \mathrm{~nm}$.

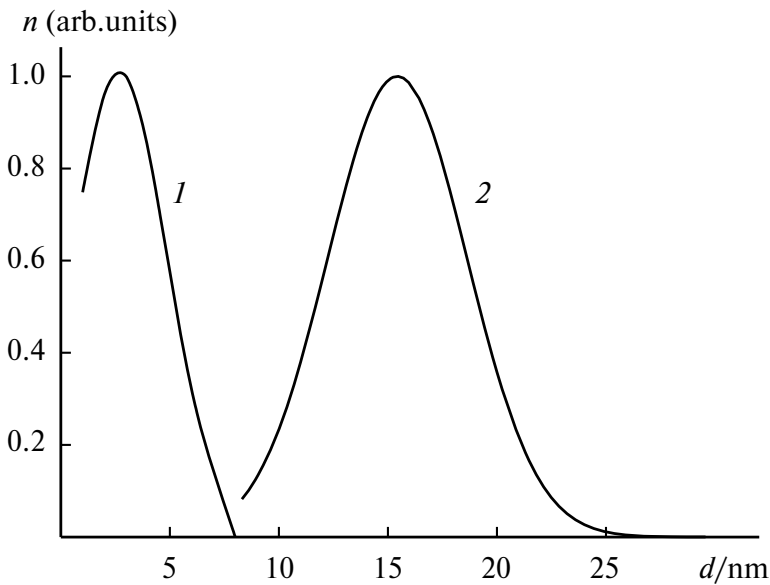

Fig. 1. The size $(d)$ distribution of the proportion $(n)$ of $\mathrm{CdS}$ cores (1) and CdS micelles (2). The approximation of experimental dependences was performed by the least squares method using the normal distribution function (Gauss distribution). CdS core size distribution obtained based on the analysis of the sc-AFM images of the dry particle cores isolated by centrifugation of the stable solution (1) and CdS micelle size distribution obtained by the dynamic light scattering directly in the stable aqueous solution (2).

The X-ray diffraction spectrum of powdered CdS nanoparticles obtained by centrifugation of the stable solution with subsequent drying is shown in Fig. 3. The positions of reflections shown by vertical lines correspond to the disordered close-packed CdS structure with the space group P6 (see Ref. 28) and the lattice parameters $a=238$ and $c=330 \mathrm{pm}$. The parameter $c$ of the nanoparticles appeared to be much smaller than the corresponding parameter of the large particles $(c=337 \mathrm{pm})$. The profile analysis of the three main reflections of this structure, namely, (001), (100), and (101), allowed us to accurately mea-

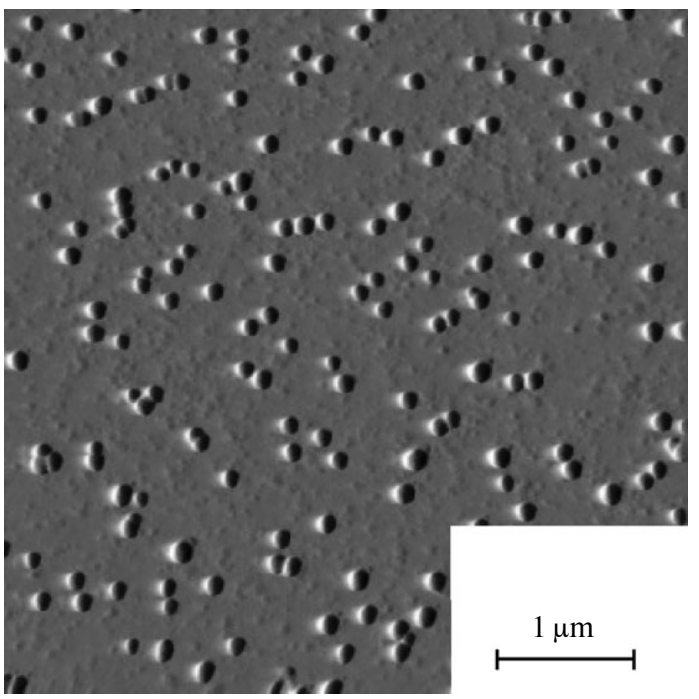

Fig. 2. Dry CdS cores (nanoparticles with the shell) on the support surface after centrifugation of the stable solution. The surface relief was measured by sc-AFM. 


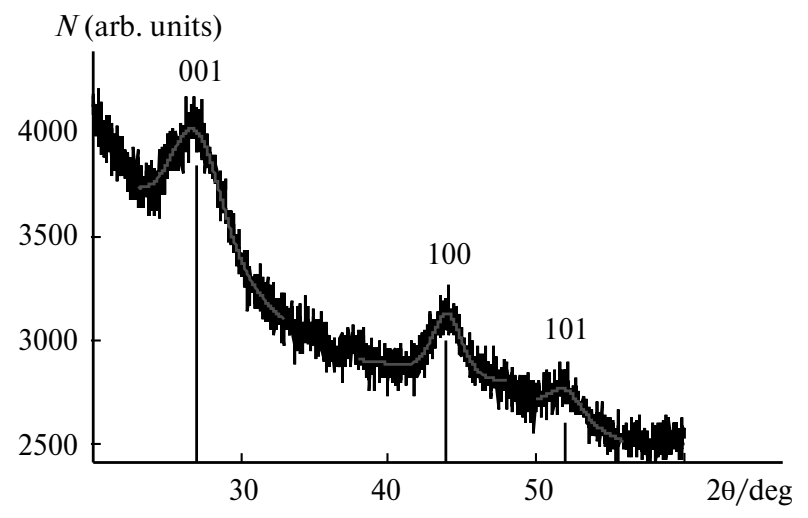

Fig. 3. X-ray diffraction spectrum of the powdered dry CdS nanoparticles obtained by centrifugation of the stable solution. The vertical lines show the positions of the reflections and the Miller indices of the disordered close-packed structure of $\mathrm{CdS}$ with the space group $P 6 . N$ is the number of pulses.

sure the broadening caused by the small size of the CdS particles. The size of the coherent scattering regions determined using the Scherrer relation and averaged over the three reflections was about $4 \mathrm{~nm}$ and thus confirmed the results obtained by the SANS and SAXS methods directly in the aqueous solution. Summing up, a CdS micelle aggregate is a single $\mathrm{CdS}$ nanoparticle about $3 \mathrm{~nm}$ in size.

\section{Results and Discussion}

Micelle model and the reason for its stabilization. In the present work, the aggregationally and sedimentationally stable colloidal solutions were synthesized by reactions (1) and (2). Crystalline $\mathrm{CdS}$ nanoparticles exist in the solution containing hydrated ions, viz., $\mathrm{Na}^{+}{ }_{a q}, \mathrm{H}^{+}{ }_{a q}$, edta ${ }^{n-}{ }_{a q}$, and $\mathrm{Cl}^{-}{ }_{a q}$. Since the solutions of $\mathrm{CdCl}_{2}$ and $\mathrm{Na}_{2} \mathrm{~S}$ were taken in the stoichiometric amounts, to a first approximation one can admit that the solution contains almost no free ions $\mathrm{Cd}^{2+}{ }_{a q}$ and $\mathrm{S}^{2-}{ }_{a q} \cdot{ }^{*}$ According to the classical adsorption rule, a crystal adsorbs ions that form a poorly soluble compound with the crystal ions, as well as ions capable of completing the crystal lattice of the solid phase. The solutions under consideration contain no such ions. Nonetheless, the colloidal solutions prepared appeared to be stable; they can be stored without visible changes at ambient temperature up to one year and during four years at a lower temperature $\left(\sim 4^{\circ} \mathrm{C}\right)$.

The stability of the solution is due to the following reasons. The $\mathrm{Na}^{+}, \mathrm{H}^{+}$, and $\mathrm{Cl}^{-}$ions cannot be strongly bound to the crystal surface, whereas the EDTA ions present in the solution can form strong complexes with the surface $\mathrm{Cd}^{2+}$ ions belonging to the crystal. In aqueous solutions, $\mathrm{H}_{4}$ edta dissociates with the formation of anions and the qualitative and quantitative ionic composition of

\footnotetext{
* Further, the subscripts " $a q$ " are omitted in the ion formulas.
}

the $\mathrm{H}_{4}$ edta solution is determined by the $\mathrm{pH}$ value. For the stable colloidal solutions prepared in the present work with the starting concentration and for those diluted tenfold, the $\mathrm{pH}$ value was 2.9 and 3.6, respectively. In this range of $\mathrm{pH}$ values, $\mathrm{H}_{4}$ edta exists in the form of $\mathrm{H}_{2} \mathrm{edta}^{2-}$ and $\mathrm{H}_{3} \mathrm{edta}^{-}$ions. The reaction of these ions with $\mathrm{Cd}^{2+}$ ions of the micelle aggregates leads to formation of mononuclear normal and protonated complexonates ${ }^{29}$ with the instability constant indices equal to $\mathrm{p} K\left(\mathrm{CdHedta}^{-}\right)=9.1$ and $\mathrm{p} K\left(\mathrm{Cdedta}^{2-}\right)=16.59$. The $\mathrm{CdHedta}^{-}$and $\mathrm{Cdedta}^{2-}$ ions coordinate and arrange the polar water molecules around themselves, thus developing an additional solvation (hydration) shell. This results in the formation of hydrophilic shells and the emergence of the adsorptionsolvation factor of the colloidal solution stability. ${ }^{\mathbf{3 0}}$

However, the formation of such strong complexonates leads to activation of disintegration (dissolution) processes of the dispersed phases formed by the CdS nanoparticles. As a result, the formation and growth of the micelle aggregate, i.e., $\mathrm{CdS}$ nanoparticles, by reaction (2) greatly decelerates and the equilibrium of this reaction shifts to the left. In discussing the micelle formula, it should be taken into account that the EDTA ions, being adsorbed on the CdS nanoparticle with the formation of $\mathrm{CdHedta}^{-}$ and $\mathrm{Cdedta}^{2-}$ ions, impose an excess negative charge on them. To sum up, the presence of the $\mathrm{H}_{2} \mathrm{edta}^{2-}$ and $\mathrm{H}_{3} \mathrm{edta}^{-}$ions promotes two parallel reactions, complexation and dissolution of the dispersed phase. Supposedly, the micelle formula can be depicted as follows:

$$
\begin{aligned}
\left\{[\mathrm{CdS}]_{m} \cdot 2 n \text { Cdedta }^{2-} \cdot(2 n-x) \mathrm{Na}^{+}{ }_{a q}\right. & \\
\left.\cdot(2 n-x) \mathrm{H}^{+}{ }_{a q}\right\}^{2 x-} \cdot x \mathrm{Na}^{+}{ }_{a q} \cdot x \mathrm{H}^{+} &
\end{aligned}
$$

where $m=1800, x<2 n$.

The complexonates adsorbing on the surface of $\mathrm{CdS}$ nanoparticles and imposing a negative charge on the micelle cores interfere with the "approach" of the $\mathrm{S}^{2-}$ anions to the cores, thus hindering the aggregation of the nanoparticles and the growth of the CdS crystal. Thus, the CdS nanoparticles with the anions adsorbed on them become aggregationally stable. It is seen from formula (3) that the micelle contains the $\mathrm{CdS}$ aggregate, which includes one CdS nanoparticle, the layer of the potential-determining ions (organic shell), and the adsorption and diffusion layers of the hydrated counterions (solvation shell).

The in situ synchrotron radiation measurements show that the initially formed CdS nanoparticle nuclei $\sim 3 \mathrm{~nm}$ in size within several dozen milliseconds remain stable (no growth and coagulation occurs) over a long period of time. The organic shell composed of the Cdedta ${ }^{2-}$ anions surrounding the $\mathrm{CdS}$ nanoparticles is about $1 \mathrm{~nm}$ thick and stable enough and disintegrates only partially upon centrifugation of the solution. This is confirmed by the formation of stable colloidal solution upon redissolu- 
tion of a centrifuged dispersed medium in distilled water. Besides, this shell, imposing an electrical charge on the nanoparticle, is also responsible for the stabilization of CdS nanoparticles. This shell is loose enough, since it does not crystallize after being dried. This was confirmed by the X-ray diffraction spectra of the "dry" nanoparticles after centrifugation and drying, which showed no additional structural lines. In turn, the organic shell is surrounded by a thick solvation shell (its thickness is $\sim 5 \mathrm{~nm}$ ), which is formed due to the negative charge on the organic shell. The solvation shell together with the organic shell and the core completes the micelle formation (Fig. 4). The solvation shell not only neutralizes the electrical charge, but also considerably decreases the mobility of the cadmium sulfide nanoparticles in solution, thus decelerating their coagulation. Taking into account that the DLS method allows one to determine not the sizes of the nanoparticles themselves, but the sizes of the moving scattering centers, which in the colloidal solution are represented by micelles, a conclusion can be drawn that the average size of the micelle described by the formula (3) in aqueous colloidal CdS solution obtained by reactions (1) and (2) is equal to $15 \mathrm{~nm}$.

In the present work, we found that the solutions of CdS nanoparticles are stable in water at the EDTA concentrations approaching the stoichiometric ratio of the cadmium and EDTA ions. The zeta-potential of the hybrid organo-inorganic cores in the solution at ambient temperature is negative; its numerical value confirms the stability of the solution. The measurements of the size and size distribution of the CdS micelles directly in the stable solution showed that the micelle aggregate consisted of the CdS nanoparticle and had an average diameter of $\sim 3 \mathrm{~nm}$, the organic shell was about $1 \mathrm{~nm}$ thick, whereas

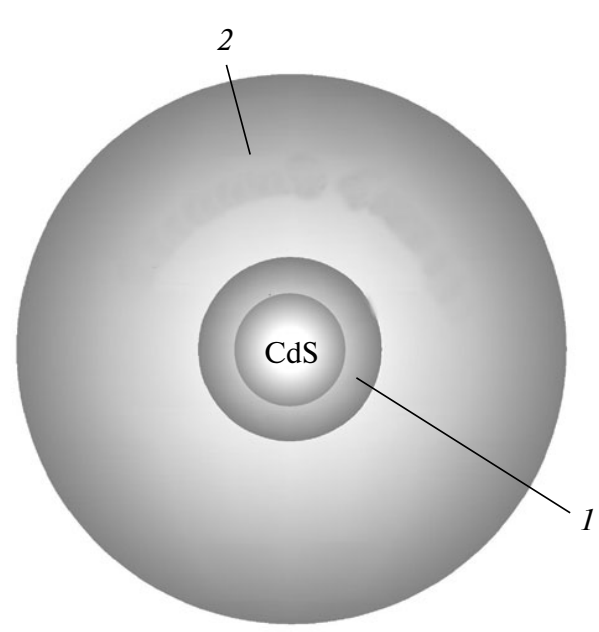

Fig. 4. Double-shell structure of the CdS micelle: the shell consisting of Cdedta ${ }^{2-}$ anions (1) and the solvation shell (2). The scaled correlation of diameters is given according to the results of measurements of diameters of the core and two shells. the solvation shell was $\sim 5 \mathrm{~nm}$ thick. Thus, the average size of the micelle in the aqueous colloidal CdS solution obtained by reactions (1) and (2) is about $15 \mathrm{~nm}$. A structural model for the double-shell CdS micelle is suggested based on the experimental data, as well as the mechanisms of its stabilization in the aqueous EDTA solution.

The authors are grateful to A. Magerl (Erlangen-Nürnberg Universität, Germany), V. Ya. Shur (Ural Federal University), and S. Yu. Bershitskii (Institute of Immunology and Physiology of the Ural Branch of the Russian Academy of Sciences) for the collaboration in performing the experiments and for discussion of the results obtained.

This work was carried out with partial financial support from the Russian Foundation for Basic Research (Project No. 12-03-00257) and the Ural Branch of the Russian Academy of Sciences (Project No. 12-P-2342003).

\section{References}

1. Y. Zhang, N. Zhang, Z.-R. Tang, Y.-J. Xu, Chem. Sci., 2012, 3, 2812.

2. V. Rajendran, M. Lehnig, C. M. Niemeyer, J. Mater. Chem., 2009, 19, 6348.

3. V. Q. Lam, S. Turrell, A. Martucci, M. Bouazaoui, B. Capoen, J. Non-Cryst. Solids, 2006, 352, 3315.

4. S. F. Wuister, A. Meijerink, J. Lumin., 2003, 102-103, 338.

5. C. Li, J. Yuan, B. Han, L. Jiang, W. Shangguan, Int. J. Hydrogen Energy, 2010, 35, 7073.

6. R. Peng, D. Zhao, J. Baltrusaitis, C.-M. Wu, R. T. Koodali, RSC Adv., 2012, 2, 5754.

7. H. Li, X. Gui, C. Ji, P. Li, Z. Li, L. Zhang, E. Shi, K. Zhu, J.Wei, K. Wang, H. Zhu, D. Wu, A. Cao, Nano Research, 2012, 5, 265.

8. J. C. Colmenares, R. Luque, J. M. Campelo, F. Colmenares, Z. Karpicski, A. A. Romero, Materials, 2009, 2, 2228.

9. H. Kisch, Adv. Photochem., 2009, 26, 93.

10. K. E. Sapsford, T. Pons, I. L. Medintz, H. Mattoussi, Sensors, 2006, 6, 925.

11. P. Sharma, S. Brown, G. Walter, S. Santra, B. Moudgil, Adv. Colloid Interface Sci., 2006, 123-126, 471.

12. X.-Y. Yu, B.-X. Lei, D.-B. Kuang, C.-Y. Su, Chem. Sci., 2011, 2, 1396.

13. V. A. Akimov, M. P. Frolov, Y. V. Korostelin, V. I. Kozlovsky, A. I. Landman, Y. P. Pomar'kov, Y. K. Skasyrsky, A. A. Voronov, Appl. Phys. B, 2009, 97, 793.

14. Y. Yin, A. P. Alivisatos, Nature, 2005, 437, 664.

15. C. L. Choi, K. J. Koski, S. Sivasankar, A. P. Alivisatos, Nano Lett., 2009, 9, 3544.

16. J. M. Bruchez, M. Moronne, P. Gin, S. Weiss, A. P. Alivisatos, Science, 1998, 281, 2013.

17. C. B. Murray, D. J. Norris, M. G. Bawendi, J. Am. Chem. Soc., 1993, 115, 8706

18. A. B. Greytak, P. M. Allen, W. Liu, J. Zhao, E. R. Young, Z. Popovic, B. J. Walker, D. G. Nocera, M. G. Bawendi, Chem. Sci., 2012, 3, 2028. 
19. J. S. Steckel, J. P. Zimmer, S. Coe-Sullivan, N. E. Stott, V. Bulovic, M. G. Bawendi, Angew. Chem., Int. Ed., 2004, 43, 2154.

20. M. A. Malik, P. O’Brien, N. Revaprasadu, J. Mater. Chem., 2001, 11, 2382.

21. H. Htoon, A. V. Malko, D. Bussian, J. Vela-Becerra, Y. Chen, J. A. Hollingsworth, V. I. Klimov, Nano Lett., 2010, 10, 2401.

22. J.-M. Moulis, F. Thévenod, Biometals, 2010, 23, 763.

23. F. Thévenod, Biometals, 2010, 23, 857.

24. P. E. Lippens, M. Lannoo, Phys. Rev. B., 1989, 39, 10935.

25. N. S. Kozhevnikova, A. S. Vorokh, A. A. Rempel', Zh. Obshch. Khim., 2010, 80, 365 [Russ. J. Gen. Chem. (Engl. Transl.), 2010, 80, 391].

26. A. A. Rempel, A. Magerl, XIX Natsion. konf. po ispol'zovaniyu sinkhrotronnogo izlucheniya SI-2012 [XIX Nation. Conf. on the Application of Synchrotron Irradiation SI-2012] (Novo- sibirsk, June 25-28, 2012), Novosibirsk, 2012, p. 121 (in Russian).

27. S. V. Rempel', A. A. Razvodov, M. S. Nebogatikov, E. V. Shishkina, V. Ya. Shur, A. A. Rempel', Fiz. Tverd. Tela, 2013, 55, 567 [Phys. Sol. State (Engl. Transl.), 2013, 55, 560].

28. A. S. Vorokh, A. A. Rempel', Dokl. Akad. Nauk, 2007, 413, 743 [Dokl. Phys. (Engl. Transl.), 2007, 52, 200].

29. Yu. Yu. Lur'e, Spravochnik po analiticheskoi khimii [Analytical Chemistry Handbook], Khimiya, Moscow, 1989, 448 pp. (in Russian).

30. S. S. Voyutskii, Kurs kolloidnoi khimii [Colloidal Chemistry Textbook], Khimiya, Moscow, 1976, 512 pp. (in Russian).

Received January 18, 2013; in revised form February 14, 2013 